\title{
A note on operator perturbation of woven frames
}

\author{
By Zhong-Qi XIANG ${ }^{*)}$ and Xiang-Chun XIAO*) \\ (Communicated by Masaki KashiwarA, M.J.A., Nov. 12, 2021)
}

\begin{abstract}
In this paper, we give a necessary and sufficient condition under which a woven frame can be preserved when applying an operator to it, which provides a correction to one existing corresponding result due to Rahimi et al. We also present some new results on the stability of woven frames under perturbation of two different operators.
\end{abstract}

Key words: Weaving frame; operator perturbation; woven frame operator; pseudoinverse.

1. Introduction. This paper adopts the following notations: $\mathscr{H}$ and $\mathscr{K}$ are complex separable Hilbert spaces, $\mathbf{J}$ is a countable index set. We use $\mathcal{B}(\mathscr{H}, \mathscr{K})$ to denote the family of bounded linear operators from $\mathscr{H}$ to $\mathscr{K}$, which, as usual, is abbreviated to $\mathcal{B}(\mathscr{H})$, if $\mathscr{K}=\mathscr{H}$. For a given number $m \in \mathbf{N}$, let $[m]=\{1,2, \cdots, m\}$, where $\mathbf{N}=$ $\{1,2, \cdots\}$. Also, the notation $\operatorname{Id}_{\mathscr{H}}$ is used to denote the identical operator on $\mathscr{H}$.

One calls a sequence $\Phi=\left\{\phi_{j}\right\}_{j \in \mathbf{J}} \subset \mathscr{H}$ a frame for $\mathscr{H}$, if there are constants $0<C_{\Phi} \leq D_{\Phi}<\infty$ such that

$$
C_{\Phi}\|f\|^{2} \leq \sum_{j \in \mathbf{J}}\left|\left\langle f, \phi_{j}\right\rangle\right|^{2} \leq D_{\Phi}\|f\|^{2}, \quad \forall f \in \mathscr{H} .
$$

The numbers $C_{\Phi}$ and $D_{\Phi}$ are called, respectively, the lower and upper frame bounds of $\Phi$.

Related to a given frame $\Phi=\left\{\phi_{j}\right\}_{j \in \mathbf{J}}$ for $\mathscr{H}$, there are two famous bounded linear operators, namely, analysis operator and frame operator, defined respectively by

$$
\begin{aligned}
& U_{\Phi}: \mathscr{H} \rightarrow \ell^{2}(\mathbf{J}), \quad U_{\Phi} f=\left\{\left\langle f, \phi_{j}\right\rangle\right\}_{j \in \mathbf{J}} . \\
& S_{\Phi}: \mathscr{H} \rightarrow \mathscr{H}, \quad S_{\Phi} f=\sum_{j \in \mathbf{J}}\left\langle f, \phi_{j}\right\rangle \phi_{j} .
\end{aligned}
$$

Recall that two frames $\Phi=\left\{\phi_{j}\right\}_{j \in \mathbf{J}}$ and $\Gamma=$ $\left\{\gamma_{j}\right\}_{j \in \mathbf{J}}$ with analysis operators $U_{\Phi}$ and $U_{\Gamma}$ respectively are said to be orthogonal, if $U_{\Phi}^{*} U_{\Gamma}=0$.

A frame in a Hilbert space is some kind of "redundant basis", which was introduced by Duffin and Schaeffer [12] in the early 1950s to investigate

2010 Mathematics Subject Classification. Primary 42C15; Secondary 42C40, 65T60.

*) School of Mathematics and Computer, Xinyu University, Xinyu, Jiangxi 338004, P. R. China.

**) School of Applied Mathematics, Xiamen University of Technology, Xiamen, Fujian 361024, P. R. China. some deep problems about nonharmonic Fourier series. Daubechies et al. [9] brought frames back to people's mind in 1986, when they discovered the close relationship between frame theory and wavelet theory. Today, frames have become to be a research hotspot because they served as an useful tool not only in theoretical work $[8,17]$, but also in dozens of application areas $[1,5,13,14,18]$.

Bemrose et al. [2] put forward a new problem in frame theory called woven frames, motivated by a question arising in distributed signal processing. Because of some potential applications such as in wireless sensor networks and pre-processing of signals, woven frames have attracted many researchers' attention $[4,6,10,19]$. And some variations of woven frames were also considered $[3,11,15,16]$.

The aim of this paper is to further study the operator perturbation of woven frames, and the motivation derives from an examination of one known result on this topic, Theorem 3.7 in [19], which stated that bounded linear operators preserve woven frames if and only if they are invertible. However, a counterexample (see Example 2.2) shows that the "only if" part of the result is not true. We provide a correction to the result (see Theorem 2.3), and some other new results on the stability of woven frames under operator perturbation are also given.

We continue with this section to collect some definitions, and basic facts on pseudo-inverse operators which will be used in next section.

Definition 1.1 (see [2]). A family of frames $\mathscr{F}=\left\{f_{i j}\right\}_{j \in \mathbf{J}, i \in[m]} \subset \mathscr{H}$ is said to be woven for $\mathscr{H}$, if there are universal constants $C_{\mathscr{F}}$ and $D_{\mathscr{F}}$ such that for any partition $\left\{\sigma_{i}\right\}_{i \in[m]}$ of $\mathbf{J}$, the family $\cup_{i \in[m]}\left\{f_{i j}\right\}_{j \in \sigma_{i}}$ is a frame for $\mathscr{H}$ with lower and upper 
frame bounds $C_{\mathscr{F}}$ and $D_{\mathscr{F}}$, respectively. Each family $\cup_{i \in[m]}\left\{f_{i j}\right\}_{j \in \sigma_{i}}$ is called a weaving.

Suppose that the family of frames $\mathscr{F}=$ $\left\{f_{i j}\right\}_{j \in \mathbf{J}, i \in[m]}$ is woven for $\mathscr{H}$. The woven frame operator associated with $\mathscr{F}$, introduced in [19], is defined by

$$
S_{\mathscr{F}}: \mathscr{H} \rightarrow \mathscr{H}, \quad S_{\mathscr{F}} f=\sum_{i \in[m]} \sum_{j \in \mathbf{J}}\left\langle f, f_{i j}\right\rangle f_{i j} .
$$

Lemma 1.2 (see [7]). Suppose that $\Theta \in$ $\mathcal{B}(\mathscr{H}, \mathscr{K})$ has closed range, then there exists a unique operator $\Theta^{\dagger} \in B(\mathscr{K}, \mathscr{H})$, called the pseudoinverse of $\Theta$, satisfying

$$
\begin{aligned}
& \Theta \Theta^{\dagger}=P_{\operatorname{Ran}(\Theta)}, \quad \Theta^{\dagger} \Theta=P_{\operatorname{Ran}\left(\Theta^{\dagger}\right)}, \\
& \left(\Theta \Theta^{\dagger}\right)^{*}=\Theta \Theta^{\dagger}, \quad\left(\Theta^{\dagger} \Theta\right)^{*}=\Theta^{\dagger} \Theta, \\
& \operatorname{Ker} \Theta^{\dagger}=\operatorname{Ran}^{\perp}(\Theta), \quad \operatorname{Ran}\left(\Theta^{\dagger}\right)=\operatorname{Ker}^{\perp} \Theta,
\end{aligned}
$$

where $P_{\operatorname{Ran}(\Theta)}$ denotes the orthogonal projection onto $\operatorname{Ran}(\Theta)$.

2. Main results. The purpose of this section is to examine the stability of woven frames under operator perturbation. We begin with the following assertion which is stated in [19] as Theorem 3.7.

Assertion 2.1. Suppose that $\mathscr{F}=$ $\left\{f_{i j}\right\}_{j \in \mathbf{J}, i \in[m]}$ is a woven frame for $\mathscr{H}$ with woven frame operator $S_{\mathscr{F}}$ and universal frame bounds $C_{\mathscr{F}}$ and $D_{\mathscr{F}}$, and that $U \in \mathcal{B}(\mathscr{H})$. Then the operator $U$ is invertible if and only if $U \mathscr{F}=\left\{U f_{i j}\right\}_{j \in \mathbf{J}, i \in[m]}$ is woven for $\mathscr{H}$. In this case, the universal frame bounds for $U \mathscr{F}$ are $C_{\mathscr{F}}\left\|U^{-1}\right\|^{-2}$ and $D_{\mathscr{F}}\|U\|^{2}$, and the woven frame operator is $U S_{\mathscr{F}} U^{*}$.

It is really true that a woven frame can be preserved by an invertible operator. But, if $U \mathscr{F}=$ $\left\{U f_{i j}\right\}_{j \in \mathbf{J}, i \in[m]}$, the operator $U$ is applied to the woven frame $\mathscr{F}=\left\{f_{i j}\right\}_{j \in \mathbf{J}, i \in[m]}$, is a woven frame, one can not conclude, in general, that $U$ is invertible. Although in the proof the authors proved that $U S_{\mathscr{F}} U^{*}$ is invertible, it does not imply that $U$ is invertible, as shown in the following example.

Example 2.2. Let $\left\{e_{j}\right\}_{j \in \mathbf{N}}$ be an orthonormal basis of $\mathscr{H}$, which, as we all known, is a frame with frame operator $S=\operatorname{Id}_{\mathscr{H}}$. We define a shift operator $U$ on $\mathscr{H}$ by $U e_{j}=e_{j-1}, j \geq 2$, and $U e_{1}=0$. Then, it is easily seen that, $U^{*}$, the adjoint operator of $U$, is given by $U^{*} f=\sum_{j \geq 2}\left\langle f, e_{j-1}\right\rangle e_{j}, f \in \mathscr{H}$. Thus

$$
\begin{aligned}
\sum_{j \in \mathbf{N}}\left|\left\langle f, U e_{j}\right\rangle\right|^{2} & =\sum_{j \in \mathbf{N}}\left|\left\langle U^{*} f, e_{j}\right\rangle\right|^{2} \\
& =\left\|U^{*} f\right\|^{2}=\sum_{j \in \mathbf{N}}\left|\left\langle f, e_{j}\right\rangle\right|^{2}=\|f\|^{2},
\end{aligned}
$$

meaning that $\left\{U e_{j}\right\}_{j \in \mathbf{N}}$ is a frame for $\mathscr{H}$. But $U$ is not invertible although

$$
\begin{aligned}
U S U^{*} f & =U U^{*} f=\sum_{j \geq 2}\left\langle f, e_{j-1}\right\rangle e_{j-1} \\
& =\sum_{j \in \mathbf{N}}\left\langle f, e_{j}\right\rangle e_{j}=f
\end{aligned}
$$

yields $U S U^{*}=\operatorname{Id} \mathscr{H}$.

Assertion 2.1 can be corrected as follows:

Theorem 2.3. Suppose that the family of frames $\mathscr{F}=\left\{f_{i j}\right\}_{j \in \mathbf{J}, i \in[m]}$ is woven for $\mathscr{H}$ with woven frame operator $S_{\mathscr{F}}$ and universal frame bounds $C_{\mathscr{F}}$ and $D_{\mathscr{F}}$, and that $U \in \mathcal{B}(\mathscr{H})$. Then $U \mathscr{F}=$ $\left\{U f_{i j}\right\}_{j \in \mathbf{J}, i \in[m]}$ is woven for $\mathscr{H}$ if and only if $U$ is surjective. In this case, the universal frame bounds and woven frame operator for $U \mathscr{F}$ are, respectively, $C_{\mathscr{F}}\left\|U^{\dagger}\right\|^{-2}$ and $D_{\mathscr{F}}\|U\|^{2}$, and $U S_{\mathscr{F}} U^{*}$. Then

Proof. " $\Leftarrow$ ". Let $\left\{\sigma_{i}\right\}_{i \in[m]}$ be any partition of $\mathbf{J}$.

$$
\begin{aligned}
\sum_{i \in[m]} \sum_{j \in \sigma_{i}}\left|\left\langle f, U f_{i j}\right\rangle\right|^{2} & =\sum_{i \in[m]} \sum_{j \in \sigma_{i}}\left|\left\langle U^{*} f, f_{i j}\right\rangle\right|^{2} \\
& \leq D_{\mathscr{F}}\left\|U^{*} f\right\|^{2} \\
& \leq D_{\mathscr{F}}\|U\|^{2}\|f\|^{2}, \quad \forall f \in \mathscr{H} .
\end{aligned}
$$

Since each $f \in \mathscr{H}$ can be expressed as $f=U g=$ $U U^{\dagger} U g=\left(U U^{\dagger}\right)^{*} U g$ for some $g \in \mathscr{H}$ by the surjectivity of $U$ and Lemma 1.2, it follows that

$$
\begin{aligned}
\|f\|^{2} & =\left\|\left(U^{\dagger}\right)^{*} U^{*} U g\right\|^{2} \leq\left\|U^{\dagger}\right\|^{2}\left\|U^{*} U g\right\|^{2} \\
& \leq \frac{\left\|U^{\dagger}\right\|^{2}}{C_{\mathscr{F}}} \sum_{i \in[m]} \sum_{j \in \sigma_{i}}\left|\left\langle U^{*} U g, f_{i j}\right\rangle\right|^{2} \\
& =\frac{\left\|U^{\dagger}\right\|^{2}}{C_{\mathscr{F}}} \sum_{i \in[m]} \sum_{j \in \sigma_{i}}\left|\left\langle U g, U f_{i j}\right\rangle\right|^{2} \\
& =\frac{\left\|U^{\dagger}\right\|^{2}}{C_{\mathscr{F}}} \sum_{i \in[m]} \sum_{j \in \sigma_{i}}\left|\left\langle f, U f_{i j}\right\rangle\right|^{2},
\end{aligned}
$$

and, consequently,

$$
C_{\mathscr{F}}\left\|U^{\dagger}\right\|^{-2}\|f\|^{2} \leq \sum_{i \in[m]} \sum_{j \in \sigma_{i}}\left|\left\langle f, U f_{i j}\right\rangle\right|^{2}, \quad \forall f \in \mathscr{H} .
$$

Altogether we know that $U \mathscr{F}$ is woven for $\mathscr{H}$ with universal frame bounds $C_{\mathscr{F}}\left\|U^{\dagger}\right\|^{-2}$ and $D_{\mathscr{F}}\|U\|^{2}$.

" $\Rightarrow$ ". As shown in the proof of Theorem 3.7 in [19], the woven frame operator of $U \mathscr{F}, S_{U \mathscr{F}}$, an 
invertible operator on $\mathscr{H}$, is given by $S_{U \mathscr{F}}=$ $U S_{\mathscr{F}} U^{*}$, implying that $U$ is surjective.

Corollary 2.4. Suppose that the family of frames $\mathscr{F}=\left\{f_{i j}\right\}_{j \in \mathbf{J}, i \in[m]}$ is woven for $\mathscr{H}$ and that $U \in \mathcal{B}(\mathscr{H})$. Then $U \mathscr{F}=\left\{U f_{i j}\right\}_{j \in \mathbf{J}, i \in[m]}$ and $U^{*} \mathscr{F}=$ $\left\{U^{*} f_{i j}\right\}_{j \in \mathbf{J}, i \in[m]}$ are both woven frames for $\mathscr{H}$ if and only if $U$ is invertible.

Proof. By Theorem 2.3,

$$
\begin{aligned}
U \mathscr{F} & =\left\{U f_{i j}\right\}_{j \in \mathbf{J}, i \in[m]} \text { is woven for } \mathscr{H} \\
& \Leftrightarrow U \text { is surjective, }
\end{aligned}
$$

and

$$
\begin{aligned}
U^{*} \mathscr{F} & =\left\{U^{*} f_{i j}\right\}_{j \in \mathbf{J}, i \in[m]} \text { is woven for } \mathscr{H} \\
& \Leftrightarrow U^{*} \text { is surjective } \Leftrightarrow U \text { is injective. }
\end{aligned}
$$

Therefore,

$$
U \mathscr{F}=\left\{U f_{i j}\right\}_{j \in \mathbf{J}, i \in[m]} \text { and } U^{*} \mathscr{F}=\left\{U^{*} f_{i j}\right\}_{j \in \mathbf{J}, i \in[m]}
$$

are both woven for $\mathscr{H} \Leftrightarrow U$ is invertible.

Corollary 2.5. Suppose that $\mathscr{F}=$ $\left\{f_{i j}\right\}_{j \in \mathbf{J}, i \in[m]}$ is a family in $\mathscr{H}$, that $\sum_{i \in[m]} \sum_{j \in \mathbf{J}}^{\langle f}\left\langle f, f_{i j}\right\rangle f_{i j}$ converges for all $f \in \mathscr{H}$, and that $U \in \mathcal{B}(\mathscr{H})$. If $U \mathscr{F}=\left\{U f_{i j}\right\}_{j \in \mathbf{J}, i \in[m]}$ and $U^{*} \mathscr{F}=\left\{U^{*} f_{i j}\right\}_{j \in \mathbf{J}, i \in[m]}$ are woven frames for $\mathscr{H}$, then $\mathscr{F}$ is a woven frame for $\mathscr{H}$.

Proof. Let us define

$$
S: \mathscr{H} \rightarrow \mathscr{H}, \quad S f=\sum_{i \in[m]} \sum_{j \in \mathbf{J}}\left\langle f, f_{i j}\right\rangle f_{i j} .
$$

If we denote by $S_{U \mathscr{F}}$, the woven frame operator of $U \mathscr{F}$, then it is easy to check that $S_{U \mathscr{F}}=U S U^{*}$. From which we conclude that $U$ is surjective. A similar discussion can show that $U^{*}$ is also surjective. Hence $U$ is invertible. Now the result follows from Theorem 2.3, since $\mathscr{F}=\left\{f_{i j}\right\}_{j \in \mathbf{J}, i \in[m]}=$ $\left\{U^{-1} U f_{i j}\right\}_{j \in \mathbf{J}, i \in[m]}$.

The following two results present more conditions for the sum of a woven frame and its perturbation under an operator to be still a woven frame.

Proposition 2.6. Suppose that the family of frames $\mathscr{F}=\left\{f_{i j}\right\}_{j \in \mathbf{J}, i \in[m]}$ is woven for $\mathscr{H}$ with universal frame bounds $C_{\mathscr{F}}$ and $D_{\mathscr{F}}$, and that $U \in$ $\mathcal{B}(\mathscr{H})$. If $U$ is a positive operator, then $\mathscr{F}+U \mathscr{F}$ is woven for $\mathscr{H}$ with universal frame bounds $C_{\mathscr{F}}$ and $D_{\mathscr{F}}\left\|\operatorname{Id}_{\mathscr{H}}+U\right\|^{2}$.

Proof. For any partition $\left\{\sigma_{i}\right\}_{i \in[m]}$ of $\mathbf{J}$ and any $f \in \mathscr{H}$ we obtain

$$
\begin{aligned}
& \sum_{i \in[m]} \sum_{j \in \sigma_{i}}\left|\left\langle f, f_{i j}+U f_{i j}\right\rangle\right|^{2} \\
& \quad=\sum_{i \in[m]} \sum_{j \in \sigma_{i}}\left|\left\langle\left(\operatorname{Id} \operatorname{H}_{\mathscr{H}}+U\right)^{*} f, f_{i j}\right\rangle\right|^{2} \\
& \quad \leq D_{\mathscr{F}}\|\operatorname{Id} \mathscr{H}+U\|^{2}\|f\|^{2}, \quad \forall f \in \mathscr{H} .
\end{aligned}
$$

Now, noting that $U$ is positive, we get

$$
\begin{aligned}
\sum_{i \in[m]} & \sum_{j \in \sigma_{i}}\left|\left\langle f, f_{i j}+U f_{i j}\right\rangle\right|^{2} \\
& \geq C_{\mathscr{F}}\left\|\left(\operatorname{Id}_{\mathscr{H}}+U\right)^{*} f\right\|^{2} \\
\quad & =C_{\mathscr{F}}\left(\|f\|^{2}+2 \operatorname{Re}\langle U f, f\rangle+\left\|U^{*} f\right\|^{2}\right) \\
& \geq C_{\mathscr{F}}\|f\|^{2},
\end{aligned}
$$

and we arrive at the result.

Theorem 2.7. Suppose that the family of frames $\mathscr{F}=\left\{f_{i j}\right\}_{j \in \mathbf{J}, i \in[m]}$ is woven for $\mathscr{H}$ with universal frame bounds $C_{\mathscr{F}}$ and $D_{\mathscr{F}}$, and that $U \in \mathcal{B}(\mathscr{H})$. If the composition of $U$ and the frame operator of each weaving is positive, then $\mathscr{F}+U \mathscr{F}$ is woven for $\mathscr{H}$ with universal frame bounds $C_{\mathscr{F}}$ and $D_{\mathscr{F}}\left\|\operatorname{Id}_{\mathscr{H}}+U\right\|^{2}$.

Proof. Let $\left\{\sigma_{i}\right\}_{i \in[m]}$ be any partition of $\mathbf{J}$. Since the universal upper frame bound is always given, we only need to show the universal lower frame bound condition. Denote by $S_{\mathscr{F}}^{\sigma}$ and $S_{\mathscr{F}+U \mathscr{F}}^{\sigma}$ be respectively the frame operators of $\cup_{i \in[m]}\left\{f_{i j}\right\}_{j \in \sigma_{i}}$ and $\cup_{i \in[m]}\left\{f_{i j}+U f_{i j}\right\}_{j \in \sigma_{i}}$. The assumption tells us that $U S_{\mathscr{F}}^{\sigma}$ is positive, then so is $S_{\mathscr{F}}^{\sigma} U^{*}$. Therefore,

$$
\begin{aligned}
S_{\mathscr{F}+U \mathscr{F}}^{\sigma} f & =\sum_{i \in[m]} \sum_{j \in \sigma_{i}}\left\langle f, f_{i j}+U f_{i j}\right\rangle\left(f_{i j}+U f_{i j}\right) \\
= & \sum_{i \in[m]} \sum_{j \in \sigma_{i}}\left\langle f, f_{i j}\right\rangle f_{i j}+U \sum_{i \in[m]} \sum_{j \in \sigma_{i}}\left\langle f, f_{i j}\right\rangle f_{i j} \\
& +\sum_{i \in[m]} \sum_{j \in \sigma_{i}}\left\langle U^{*} f, f_{i j}\right\rangle f_{i j} \\
& +U \sum_{i \in[m]} \sum_{j \in \sigma_{i}}\left\langle U^{*} f, f_{i j}\right\rangle f_{i j} \\
= & S_{\mathscr{F}}^{\sigma} f+U S_{\mathscr{F}}^{\sigma}+S_{\mathscr{F}}^{\sigma} U^{*} f+U S_{\mathscr{F}}^{\sigma} U^{*} f \\
\geq & S_{\mathscr{F}}^{\sigma} f
\end{aligned}
$$

for each $f \in \mathscr{H}$. This leads to

$$
\begin{aligned}
\sum_{i \in[m]} \sum_{j \in \sigma_{i}}\left|\left\langle f, f_{i j}+U f_{i j}\right\rangle\right|^{2} & =\left\langle S_{\mathscr{\mathscr { F }}+U \mathscr{F}}^{\sigma} f, f\right\rangle \\
& \geq\left\langle S_{\mathscr{F}}^{\sigma} f, f\right\rangle \geq C_{\mathscr{F}}\|f\|^{2},
\end{aligned}
$$

as desired.

The following operator perturbation type for 
woven frames is more general, comparing to the ones in Proposition 2.6 and Theorem 2.7.

Theorem 2.8. Suppose that $\mathscr{F}=$ $\left\{f_{i j}\right\}_{j \in \mathbf{J}, i \in[m]}$ and $\mathscr{G}=\left\{g_{i j}\right\}_{j \in \mathbf{J}, i \in[m]}$ are woven frames for $\mathscr{H}$ with universal frame bounds $C_{\mathscr{F}}, D_{\mathscr{F}}$ and $C_{\mathscr{G}}, D_{\mathscr{G}}$ respectively, and that $T_{1}, T_{2} \in \mathcal{B}(\mathscr{H})$. If for any partition of $\mathbf{J}$, the two weaving frames generated respectively by $\mathscr{F}$ and $\mathscr{G}$ are orthogonal, and $T_{1}$ or $T_{2}$ is surjective, then $T_{1} \mathscr{F}+T_{2} \mathscr{F}$ is woven for $\mathscr{H}$.

Proof. For any given partition $\left\{\sigma_{i}\right\}_{i \in[m]}$ of $\mathbf{J}$, let $U_{\mathscr{F}}^{\sigma}$ and $U_{\mathscr{G}}^{\sigma}$ be, respectively, the analysis operators of $\cup_{i \in[m]}\left\{f_{i j}\right\}_{j \in \sigma_{i}}$ and $\cup_{i \in[m]}\left\{g_{i j}\right\}_{j \in \sigma_{i}}$. We assume, without loss of generality, that $T_{1}$ is surjective. Then, by the same way as that presented in the proof of Theorem 2.3 we can show that $\left\|T_{1}^{*} f\right\|^{2} \geq\left\|T_{1}^{\dagger}\right\|^{-2}\|f\|^{2}$ for each $f \in \mathscr{H}$. Noting also that $\left(U_{\mathscr{F}}^{\sigma}\right)^{*} U_{\mathscr{G}}^{\sigma}=\left(U_{\mathscr{G}}^{\sigma}\right)^{*} U_{\mathscr{F}}^{\sigma}=0$, we have

$$
\begin{aligned}
\sum_{i \in[m]} & \sum_{j \in \sigma_{i}}\left|\left\langle f, T_{1} f_{i j}+T_{2} g_{i j}\right\rangle\right|^{2} \\
= & \left\|U_{\mathscr{F}}^{\sigma} T_{1}^{*} f+U_{\mathscr{G}}^{\sigma} T_{2}^{*} f\right\|^{2} \\
= & \left\langle\left(U_{\mathscr{F}}^{\sigma} T_{1}^{*}+U_{\mathscr{G}}^{\sigma} T_{2}^{*}\right)^{*}\left(U_{\mathscr{F}}^{\sigma} T_{1}^{*}+U_{\mathscr{G}}^{\sigma} T_{2}^{*}\right) f, f\right\rangle \\
= & \left\langle T_{1}\left(U_{\mathscr{F}}^{\sigma}\right)^{*} U_{\mathscr{F}}^{\sigma} T_{1}^{*} f, f\right\rangle \\
& +\left\langle T_{1}\left(U_{\mathscr{F}}^{\sigma}\right)^{*} U_{\mathscr{G}}^{\sigma} T_{2}^{*} f, f\right\rangle \\
& +\left\langle T_{2}\left(U_{\mathscr{G}}^{\sigma}\right)^{*} U_{\mathscr{F}}^{\sigma} T_{1}^{*} f, f\right\rangle \\
& +\left\langle T_{2}\left(U_{\mathscr{G}}^{\sigma}\right)^{*} U_{\mathscr{G}}^{\sigma} T_{2}^{*} f, f\right\rangle \\
= & \left\langle\left(U_{\mathscr{F}}^{\sigma}\right)^{*} U_{\mathscr{F}}^{\sigma} T_{1}^{*} f, T_{1}^{*} f\right\rangle+\left\langle\left(U_{\mathscr{G}}^{\sigma}\right)^{*} U_{\mathscr{G}}^{\sigma} T_{2}^{*} f, T_{2}^{*} f\right\rangle \\
\geq & \left\langle\left(U_{\mathscr{F}}^{\sigma}\right)^{*} U_{\mathscr{F}}^{\sigma} T_{1}^{*} f, T_{1}^{*} f\right\rangle \\
= & \sum_{i \in[m]} \sum_{j \in \sigma_{i}}\left|\left\langle T_{1}^{*} f, f_{i j}\right\rangle\right|^{2} \\
\geq & C_{\mathscr{F}}\left\|T_{1}^{*} f\right\|^{2} \geq C_{\mathscr{F}}\left\|T_{1}^{\dagger}\right\|^{-2}\|f\|^{2}
\end{aligned}
$$

for each $f \in \mathscr{H}$. It is trivial to show that

$$
\begin{aligned}
\sum_{i \in[m]} & \sum_{j \in \sigma_{i}}\left|\left\langle f, T_{1} f_{i j}+T_{2} g_{i j}\right\rangle\right|^{2} \\
= & \left\|U_{\mathscr{F}}^{\sigma} T_{1}^{*} f+U_{\mathscr{G}}^{\sigma} T_{2}^{*} f\right\|^{2} \\
\leq & 2\left(\left\|U_{\mathscr{F}}^{\sigma} T_{1}^{*} f\right\|^{2}+\left\|U_{\mathscr{G}}^{\sigma} T_{2}^{*} f\right\|^{2}\right) \\
= & 2\left(\sum_{i \in[m]} \sum_{j \in \sigma_{i}}\left|\left\langle T_{1}^{*} f, f_{i j}\right\rangle\right|^{2}\right. \\
& \left.+\sum_{i \in[m]} \sum_{j \in \sigma_{i}}\left|\left\langle T_{2}^{*} f, g_{i j}\right\rangle\right|^{2}\right) \\
\leq & 2\left(D_{\mathscr{F}}\left\|T_{1}^{*} f\right\|^{2}+D_{\mathscr{G}}\left\|T_{2}^{*} f\right\|^{2}\right) \\
\leq & 2\left(D_{\mathscr{F}}\left\|T_{1}\right\|^{2}+D_{\mathscr{G}}\left\|T_{2}\right\|^{2}\right)\|f\|^{2}
\end{aligned}
$$

and the proof is completed.
An alternative condition under which the conclusion of Theorem 2.8 remains true is given below.

Theorem 2.9. Suppose that $\mathscr{F}=$ $\left\{f_{i j}\right\}_{j \in \mathbf{J}, i \in[m]}$ and $\mathscr{G}=\left\{g_{i j}\right\}_{j \in \mathbf{J}, i \in[m]}$ are woven frames for $\mathscr{H}$ with universal frame bounds $C_{\mathscr{F}}, D_{\mathscr{F}}$ and $C_{\mathscr{G}}, D_{\mathscr{G}}$ respectively, and that $T_{1}, T_{2} \in \mathcal{B}(\mathscr{H})$. If $T_{1}$ is surjective with $\frac{1}{2}\left\|T_{1}^{\dagger}\right\|^{-2} C_{\mathscr{F}}-D_{\mathscr{G}}\left\|T_{2}\right\|^{2}>0$ or $T_{2}$ is surjective with $\frac{1}{2}\left\|T_{2}^{\dagger}\right\|^{-2} C_{\mathscr{G}}-D_{\mathscr{F}}\left\|T_{1}\right\|^{2}>0$, then $T_{1} \mathscr{F}+T_{2} \mathscr{F}$ is woven for $\mathscr{H}$.

Proof. Without loss of generality, let $T_{1}$ be surjective with $\frac{1}{2}\left\|T_{1}^{\dagger}\right\|^{-2} C_{\mathscr{F}}-D_{\mathscr{G}}\left\|T_{2}\right\|^{2}>0$. For any partition $\left\{\sigma_{i}\right\}_{i \in[m]}$ of $\mathbf{J}$ and any $f \in \mathscr{H}$ we have

$$
\begin{aligned}
& \sum_{i \in[m]} \sum_{j \in \sigma_{i}}\left|\left\langle f, T_{1} f_{i j}\right\rangle\right|^{2} \\
& =\sum_{i \in[m]} \sum_{j \in \sigma_{i}}\left|\left\langle f, T_{1} f_{i j}+T_{2} g_{i j}-T_{2} g_{i j}\right\rangle\right|^{2} \\
& \leq 2\left(\sum_{i \in[m]} \sum_{j \in \sigma_{i}}\left|\left\langle f, T_{1} f_{i j}+T_{2} g_{i j}\right\rangle\right|^{2}\right. \\
& \left.\quad+\sum_{i \in[m]} \sum_{j \in \sigma_{i}}\left|\left\langle f, T_{2} g_{i j}\right\rangle\right|^{2}\right) .
\end{aligned}
$$

Therefore,

$$
\begin{aligned}
\sum_{i \in[m]} & \sum_{j \in \sigma_{i}}\left|\left\langle f, T_{1} f_{i j}+T_{2} g_{i j}\right\rangle\right|^{2} \\
\geq & \frac{1}{2} \sum_{i \in[m]} \sum_{j \in \sigma_{i}}\left|\left\langle f, T_{1} f_{i j}\right\rangle\right|^{2} \\
& -\sum_{i \in[m]} \sum_{j \in \sigma_{i}}\left|\left\langle f, T_{2} g_{i j}\right\rangle\right|^{2} \\
\geq & \frac{1}{2} C_{\mathscr{F}}\left\|T_{1}^{*} f\right\|^{2}-D_{\mathscr{G}}\left\|T_{2}^{*} f\right\|^{2} \\
\geq & \frac{1}{2} C_{\mathscr{F}}\left\|T_{1}^{\dagger}\right\|^{-2}\|f\|^{2}-D_{\mathscr{G}}\left\|T_{2}\right\|^{2}\|f\|^{2} \\
= & \left(\frac{1}{2} C_{\mathscr{F}}\left\|T_{1}^{\dagger}\right\|^{-2}-D_{\mathscr{G}}\left\|T_{2}\right\|^{2}\right)\|f\|^{2} .
\end{aligned}
$$

The universal upper frame bound condition is straightforward, or check the second part, proof of Theorem 2.8.

In [2, Example 2], the authors told us that applying two different operators to woven frames can give frames that are not woven. So a natural problem arises: To make woven frames to be still woven when applying two different operators to them, what conditions need to be attached? To this problem, we have the following 
Theorem 2.10. Suppose the frames $\mathscr{F}^{\prime}=$ $\left\{f_{j}\right\}_{j \in \mathbf{J}}$ and $\mathscr{G}^{\prime}=\left\{g_{j}\right\}_{j \in \mathbf{J}}$ with frame bounds $C_{\mathscr{F}^{\prime}}, D_{\mathscr{F}^{\prime}}$ and $C_{\mathscr{G}^{\prime}}, D_{\mathscr{G}^{\prime}}$ respectively are woven for $\mathscr{H}$ with universal frame bounds $C_{\mathscr{F}^{\prime} \mathscr{G}^{\prime}}$ and $D_{\mathscr{F}^{\prime} \mathscr{G}^{\prime}}$, and that $T_{1}, T_{2} \in \mathcal{B}(\mathscr{H})$. If $T_{1}$ is surjective and $\sqrt{C_{\mathscr{F}^{\prime} \mathscr{G}^{\prime}}}\left\|T_{1}^{\dagger}\right\|^{-1}>\sqrt{D_{\mathscr{G}}}\left\|T_{1}-T_{2}\right\|$, then $T_{1} \mathscr{F}^{\prime}$ and $T_{2} \mathscr{G}^{\prime}$ are woven for $\mathscr{H}$ with universal frame bounds $\quad\left(\sqrt{C_{\mathscr{F}^{\prime} \mathscr{G}^{\prime}}}\left\|T_{1}^{\dagger}\right\|^{-1}-\sqrt{D_{\mathscr{G}^{\prime}}}\left\|T_{1}-T_{2}\right\|\right)^{2} \quad$ and $\left(D_{\mathscr{F}^{\prime}}\left\|T_{1}\right\|^{2}+D_{\mathscr{G}_{1}}\left\|T_{2}\right\|^{2}\right)$.

Proof. Let $\left\{\sigma_{1}, \sigma_{2}\right\}$ be any partition of $\mathbf{J}$. The universal upper frame bound condition follows from the following calculation:

$$
\begin{aligned}
& \sum_{j \in \sigma_{1}}\left|\left\langle f, T_{1} f_{j}\right\rangle\right|^{2}+\sum_{j \in \sigma_{2}}\left|\left\langle f, T_{2} g_{j}\right\rangle\right|^{2} \\
& \quad=\sum_{j \in \sigma_{1}}\left|\left\langle T_{1}^{*} f, f_{j}\right\rangle\right|^{2}+\sum_{j \in \sigma_{2}}\left|\left\langle T_{2}^{*} f, g_{j}\right\rangle\right|^{2} \\
& \quad \leq \sum_{j \in \mathbf{J}}\left|\left\langle f, T_{1} f_{j}\right\rangle\right|^{2}+\sum_{j \in \mathbf{J}}\left|\left\langle f, T_{2} g_{j}\right\rangle\right|^{2} \\
& \quad \leq D_{\mathscr{F}^{\prime}}\left\|T_{1}^{*} f\right\|^{2}+D_{\mathscr{G}^{\prime}}\left\|T_{2}^{*} f\right\|^{2} \\
& \quad \leq\left(D_{\mathscr{F}^{\prime}}\left\|T_{1}\right\|^{2}+D_{\mathscr{G}^{\prime}}\left\|T_{2}\right\|^{2}\right)\|f\|^{2}, \quad \forall f \in \mathscr{H} .
\end{aligned}
$$

For the universal lower frame bound condition, we have by Minkowski's inequality and subadditivity of the square root function that

$$
\begin{aligned}
& \left(\sum_{j \in \sigma_{1}}\left|\left\langle f, T_{1} f_{j}\right\rangle\right|^{2}+\sum_{j \in \sigma_{2}}\left|\left\langle f, T_{2} g_{j}\right\rangle\right|^{2}\right)^{\frac{1}{2}} \\
& =\left(\sum_{j \in \sigma_{1}}\left|\left\langle T_{1}^{*} f, f_{j}\right\rangle\right|^{2}\right. \\
& \left.+\sum_{j \in \sigma_{2}}\left|\left\langle T_{1}^{*} f+\left(T_{2}^{*}-T_{1}^{*}\right) f, g_{j}\right\rangle\right|^{2}\right)^{\frac{1}{2}} \\
& \geq\left(\sum_{j \in \sigma_{1}}\left|\left\langle T_{1}^{*} f, f_{j}\right\rangle\right|^{2}+\sum_{j \in \sigma_{2}}\left|\left\langle T_{1}^{*} f, g_{j}\right\rangle\right|^{2}\right)^{\frac{1}{2}} \\
& -\left(\sum_{j \in \sigma_{2}}\left|\left\langle\left(T_{2}^{*}-T_{1}^{*}\right) f, g_{j}\right\rangle\right|^{2}\right)^{\frac{1}{2}} \\
& \geq \sqrt{C_{\mathscr{F}^{\prime} \boldsymbol{G}^{\prime}}}\left\|T_{1}^{*} f\right\|-\left(\sum_{j \in \mathbf{J}}\left|\left\langle\left(T_{2}^{*}-T_{1}^{*}\right) f, g_{j}\right\rangle\right|^{2}\right)^{\frac{1}{2}} \\
& \geq \sqrt{C_{\mathscr{F}^{\prime} \mathscr{G}_{\prime}^{\prime}}}\left\|T_{1}^{\dagger}\right\|^{-1}\|f\|-\sqrt{D_{\mathscr{G}^{\prime}}}\left\|T_{1}-T_{2}\right\|\|f\| \\
& =\left(\sqrt{C_{\mathscr{F} \mathscr{G}^{\prime}}}\left\|T_{1}^{\dagger}\right\|^{-1}-\sqrt{D_{\mathscr{G}}}\left\|T_{1}-T_{2}\right\|\right)\|f\|,
\end{aligned}
$$

where in the last inequality we apply the fact that $\left\|T_{1}^{*} f\right\| \geq\left\|T_{1}^{\dagger}\right\|^{-1}\|f\|$ for any $f \in \mathscr{H}$, and the proof is finished.
Corollary 2.11. Suppose that $\mathscr{F}^{\prime}=\left\{f_{j}\right\}_{j \in \mathbf{J}}$ is a frame for $\mathscr{H}$ with frame bounds $C_{\mathscr{F}^{\prime}}$ and $D_{\mathscr{F}^{\prime}}$, and that $T_{1}, T_{2} \in \mathcal{B}(\mathscr{H})$. If $T_{1}$ is surjective and $\sqrt{C_{\mathscr{F ^ { \prime }}}}\left\|T_{1}^{\dagger}\right\|^{-1}>\sqrt{D_{\mathscr{F ^ { \prime }}}}\left\|T_{1}-T_{2}\right\|$, then $T_{1} \mathscr{F}^{\prime}$ and $T_{2} \mathscr{F}^{\prime}$ are woven for $\mathscr{H}$ with universal frame bounds $\left(\sqrt{C_{\mathscr{F}^{\prime}}}\left\|T_{1}^{\dagger}\right\|^{-1}-\sqrt{D_{\mathscr{F}^{\prime}}}\left\|T_{1}-T_{2}\right\|\right)^{2}$ and $D_{\mathscr{F}^{\prime}}\left(\left\|T_{1}\right\|^{2}+\right.$ $\left.\left\|T_{2}\right\|^{2}\right)$.

Proof. Since $\mathscr{F}^{\prime}$ is woven to itself, the result follows immediately from Theorem 2.10.

Corollary 2.12. Suppose that $\mathscr{F}^{\prime}=\left\{f_{j}\right\}_{j \in \mathbf{J}}$ is a frame for $\mathscr{H}$ with frame bounds $C_{\mathscr{F} \prime}$ and $D_{\mathscr{F}}$, and that $T \in \mathcal{B}(\mathscr{H})$. If $\left\|\operatorname{Id}_{\mathscr{H}}-T\right\|<\sqrt{\frac{C_{\mathscr{F}}}{D_{\mathscr{P}^{\prime}}}}$, then $\mathscr{F}^{\prime}$ and $T \mathscr{F}^{\prime}$ are woven for $\mathscr{H}$.

Proof. The result follows if in Corollary 2.11 we take $T_{1}=\operatorname{Id}_{\mathscr{H}}$ and $T_{2}=T$.

Remark 2.13. Corollary 2.12 is just Proposition 6.2 in [2].

Acknowledgements. The research is supported by the Science Foundation of Jiangxi Education Department (Grant Nos. GJJ202302 and GJJ190886) and the Natural Science Foundation of Fujian Province, China (Grant No. 2021J011192).

\section{References}

[ 1 ] P. Balazs, N. Holighaus, T. Necciari and D. Stoeva, Frame theory for signal processing in psychoacoustics, in Excursions in harmonic analysis. Vol. 5, Appl. Numer. Harmon. Anal, Birkhäuser/Springer, Cham, 2017, pp. 225-268.

[ 2 ] T. Bemrose, P. G. Casazza, K. Gröchenig, M. C. Lammers and R. G. Lynch, Weaving frames, Oper. Matrices 10 (2016), no. 4, 1093-1116.

[ 3 ] A. Bhandari, D. Borah and S. Mukherjee, Characterizations of weaving $K$-frames, Proc. Japan Acad. Ser. A Math. Sci. 96 (2020), no. 5, 39-43.

[ 4 ] A. Bhandari and S. Mukherjee, Characterizations of woven frames, Int. J. Wavelets Multiresolut. Inf. Process. 18 (2020), no. 5, 2050033.

[ 5 ] H. Bölcskei, F. Hlawatsch and H. G. Feichtinger, Frame-theoretic analysis of oversampled filter banks, IEEE Trans. Signal Process. 46 (1998), 3256-3268.

[ 6 ] P. G. Casazza and R. G. Lynch, Weaving properties of Hilbert space frames, in The 2015 International Conference on Sampling Theory and Applications (SampTA 2015), 25-29 May 2015, (Washington, DC, 2015), 110-114, Proceedings of the 2015 International Conference on Sampling Theory and Applications (SampTA 2015), IEEE, New York, 2015.

[ 7 ] O. Christensen, An introduction to frames and Riesz bases, 2nd ed., Applied and Numerical Harmonic Analysis, Birkhäuser/Springer, Berlin, 2016. 
[ 8 ] F. Dai, Characterizations of function spaces on the sphere using frames, Trans. Amer. Math. Soc. 359 (2007), no. 2, 567-589.

[ 9 ] I. Daubechies, A. Grossmann and Y. Meyer, Painless nonorthogonal expansions, J. Math. Phys. 27 (1986), no. 5, 1271-1283.

[ 10 ] Deepshikha and L. K. Vashisht, On weaving frames, Houston J. Math. 44 (2018), no. 3, 887-915.

[11 ] Deepshikha and L. K. Vashisht, Weaving $K$-frames in Hilbert spaces, Results Math. 73 (2018), no. 2, Paper No. 81.

[12] R. J. Duffin and A. C. Schaeffer, A class of nonharmonic Fourier series, Trans. Amer. Math. Soc. 72 (1952), 341-366.

[13 ] H. G. Feichtinger and K. Gröchenig, Theory and practice of irregular sampling, in Wavelets: mathematics and applications, Stud. Adv. Math, CRC, Boca Raton, FL, 1994, pp. 305363.

[14] J.-P. Gazeau, Coherent States in Quantum Physics, Wiley-VCH, Berlin, 2009.
[15] A. Khosravi and J. S. Banyarani, Weaving $g$-frames and weaving fusion frames, Bull. Malays. Math. Sci. Soc. 42 (2019), no. 6, 3111-3129.

[16 ] D. Li, J. S. Leng, T. Z. Huang and X. P. Li, On weaving $g$-frames for Hilbert spaces, Complex Anal. Oper. Theory 14 (2020), no. 2, Paper No. 33.

[17 ] I. Z. Pesenson, Paley-Wiener-Schwartz nearly Parseval frames on noncompact symmetric spaces, in Commutative and noncommutative harmonic analysis and applications, Contemp. Math., 603, Amer. Math. Soc., Providence, RI, 2013, pp. 55-71.

[18 C. Poon, A consistent and stable approach to generalized sampling, J. Fourier Anal. Appl. 20 (2014), no. 5, 985-1019.

[ 19 ] A. Rahimi, Z. Samadzadeh and B. Daraby, Frame-related operators for woven frames, Int. J. Wavelets Multiresolut. Inf. Process. 17 (2019), no. 3, 1950010. 